\title{
HEURISTISCHE KONFIGURATION GUTER AUFBEREITUNGSANLAGEN FÜR BAURESTMASSEN
}

\author{
Marcus Nicolai, TH Karlsruhe \\ Thomas Spengler, TH Karlsruhe \\ Otto Rentz, TH Karlsruhe
}

Die Förderung einer rückstandsarmen Kreislaufwirtschaft im Sinne geschlossener Stoffkreisläufe ist gemäß dem Entwurf des Kreislaufwirtschafts- und Abfallgesetzes der Bundesregierung primäres Ziel zukünftiger Abfallgesetzgebung. Die Realisierung erfordert eine Verwertung von Reststoffe:l auf hoher Qualitätsstufe. Im Jahre 1989 fielen in der Bunderepublik Deutschland 22,6 Mio t Bauschutt an, lediglich 16\% hiervon wurden stofflich verwertet. Dabei wurden 528 als Tragschichten im Straßenbau, der Rest in Lärmschutzwällen, Dämmen und im Landschaftsbau eingesetzt. Auf gleicher Qualitätsstufe, etwa als Betonzuschlag, fand keine Verwertung statt.

Der Stand der Technik von Aufbereitungsanlagen für Baurestmassen ist dementsprechend durch niedrige Produktqualifäten bei geringen spezifischen Aufbereitungskosten gekennzeichnet, obwohl hohe Produktqualitäten prinzipiell erreichbar sind. Der ökonomische Rahmen der Anlagen wird durch erzielbare Annahmepreise und das Preisniveau konkurrierender konventioneller Baustoffe eingeschränkt. Zunehmend restriktive Nutzungsmöglichkeiten von Deponiekapazitäten sowie restriktivere Genehmigung des Abbaus von Naturbaustoffen führen zu steigenden Deponiekosten und Baustoffpreisen, wodurch der wirtschastliche Spielraum für die Entwicklung und implementierung aufwendigerer Aufbereitungstechniken gegeben ist. Dabei bestimmen die eingesetzten Aggregate einerseits und deren Konfiguration andererseits entscheidend die erzielbaren Produktqualitäten, Verwertungsquoten und die Aufbereitungskosten.

Ziel des Vortrags ist es, einen heuristischen Ansatz zur Konfiguration guter Aufbereitungsanlagen für Bauschutt vorzustellen. Ausgehend von einer Analyse von 30 bestehenden Anlagen in der Region Oberrhein und einer mathematischen Modellierung der technischen Aggregate wird zunächst ein auf dem Flow-Sheeting chemischer Anlagen beruhendes Simulationsmodell zur Ermittlung von Stoff- und Energiebilanzen sowie Aufbereitungskosten beliebig konfigurierter Anlagen entwickelt. Mit Hilfe eines heuristisch modifizierten Branch and Bound Verfahrens werden verschiedene Anlagenkonfigurationen erzeugt, die zugehörigen kostenminimalen Aggregatelisten erstellt und so das zugrundeliegende kombinatorische Optimierungsproblem gelöst. Die Sensivität der entwickelten "optimalen" Anlagen bezüglich Aufgabegut, Annahmepreise und Produktpreise wird untersucht. Mindestverwertungsquoten, -produktqualitäten sowie einzuhaltende Grenzwerte für Schadstoffe in den Produkten können exogen vorgegeben werden. 